\title{
MAPPING ROADWAY DRAINAGE DITCHES USING MOBILE LIDAR
}

\author{
Yi-Chun Lin ${ }^{\mathrm{a}}$, Darcy Bullock ${ }^{\mathrm{a}}$, Ayman Habib ${ }^{\mathrm{a}, *}$ \\ ${ }^{a}$ Lyles School of Civil Engineering, Purdue University, West Lafayette, IN 47907, USA - (lin934, darcy, ahabib)@ purdue.edu
}

\author{
Commission I, WG I/7
}

KEY WORDS: Mobile Mapping System, LiDAR, Roadside ditch, Point cloud, Quality control

\begin{abstract}
:
Roadside ditches serve an important role for draining storm water. Over time vegetation growth, natural sediment deposits, and other debris can change grade of ditches. Effectively monitoring and identifying these changes to prioritize ditch maintenance is important from both a pavement preservation perspective and prevention of localized flooding. This study evaluates the performance of two mobile LiDAR systems for mapping the cross-section of roadside ditches in the presence of vegetation. The geometric quality of data collected by two different wheel-based LiDAR systems were investigated. The mapped ditches were reported and visualized in 2D images as well as 3D point clouds. The cross-sections of man-made drainage ditches were extracted and the quality of mapped ditches was assessed against Real-Time Kinematic Global Navigation Satellite Systems (RTK-GNSS) survey. The overall point cloud accuracy was $4 \mathrm{~cm}$ for the medium-grade system, and $1 \mathrm{~cm}$ for the high-grade system. The mapping accuracy is $2 \mathrm{~cm}$ (medium-grade system) and $1 \mathrm{~cm}$ (high-grade system) for solid surface. For rough mowed areas and areas with significant vegetation, the vertical accuracy was found to be $7 \mathrm{~cm}$ and $11 \mathrm{~cm}$, respectively, for both wheel-based systems.
\end{abstract}

\section{INTRODUCTION}

Roadside ditches are important for conveying road runoff away from transportation throughways. When properly maintained, ditches help improve the functionality of road network by prolonging the lifespan of the pavement and preventing local flooding. Proper detection and mapping of roadside ditches is not only crucial to road maintenance, but also lays the foundation for assessing their impact on the natural hydrologic and nutrient transport network (Buchanan et al., 2013). Studies in New York State (NYS) and Ohio have pointed out the lack of maintenance of roadside ditches due to limited time, labor, equipment, and funding (Schneider et al., 2019; Matos, 2016). Schneider et al. (2019) conducted a survey that involved town and county highway professionals across NYS to determine their ditch management practices. It was estimated that one-third to one-half of the roadside ditches across upstate NYS are in fair to poor condition.

Ditch detection and mapping that makes use of high resolution LiDAR point cloud can be a cost effective alternative to field surveys for prioritizing and planning ditch maintenance. It also eliminates exposure of survey crews to the hazards of working adjacent to a roadway. This paper evaluates the feasibility of using LiDAR data acquired by Mobile Mapping Systems (MMS) for high resolution mapping of roadside ditches in the presence of vegetation. The geometric quality of the data collected by two different MMS is also investigated. Ditch cross-sections are extracted from MMS LiDAR data and their quality is assessed against Real-Time Kinematic Global Navigation Satellite Systems (RTK-GNSS) survey. A reporting mechanism that visualizes the cross-sections in 2D images as well as 3D point clouds is presented. The rest of the paper is structured as follows: a brief summary of the literature, followed by a description of the two mobile mapping systems, study area, quality assessment of the data, characterization of expected spatial accuracy of various drainage surfaces, and discussion of future applications.

\section{LITERATURE REVIEW}

Proper mapping of ditch networks is a valuable data for road maintenance as well as hydrologic modeling. Field survey, Digital Elevation Models (DEM), and airborne LiDAR are the main sources used in previous studies. Levavasseur et al. (2015) conducted exhaustive field surveys to model the spatial variability of man-made drainage density in agricultural landscapes. The study pointed out that remote sensing data may not be accurate enough to capture man-made drainage ditches, which can be very narrow (width and depth of less than $1 \mathrm{~m}$ ) and densely covered with vegetation. Rapinel et al. (2015) derived DEMs from airborne LiDAR data with different point densities, and then extracted drainage network from the DEM using an object-based image analysis approach. The result suggested that the quality of the drainage network map significantly depends on the point density of the LiDAR - which is consistent with Levavasseur et al. (2015). A pilot study for roadside ditch detection was conducted in 2017 by the Chesapeake Bay Program Partnership's (CBP) Roadside Ditch Management Team (RDMT) in Pennsylvania Ibeh et al. (2017). The study utilized a DEM generated from airborne LiDAR data, and detected roadside ditches based on three metrics: flow accumulation, positive topographic openness, and topographic convergence index. The study also recommended that a DEM be created from high-quality point cloud. The main limitation of this study is the lack of quantitative quality control - the quality of the derived outcome was assessed using visual interpretation of aerial imagery. Roelens et al. (2018a, 2018b) extracted drainage ditches directly from irregular airborne LiDAR point clouds with an average point spacing of $0.10 \mathrm{~m}$ instead of interpolated DEM. The LiDAR points were classified as ditch and non-ditch points using random forest classifier. The ditch dropout points were then reconstructed to form blind zones. Finally, the LiDAR ditch points and dropouts were assembled into ditch objects (2D-polygons and their derived centerlines). The procedure was tested in a grassland and a peri-urban agricultural area. The derived ditch centerlines had a positional accuracy of $0.5 \mathrm{~m}$.

${ }^{*}$ Corresponding author. 
Previous studies suggested that the ground sampling distance of the DEM or the inter-point spacing of the LiDAR data is critical to ensuring the quality of ditch mapping. This study utilizes wheel-based LiDAR data which has a much higher point density and accuracy when compared to airborne LiDAR data. The objectives of this study are to:

1. visualize the ditch cross-sections in $2 \mathrm{D}$ and $3 \mathrm{D}$,

2. assess the geometric quality of data collected by two grades of wheel-based LiDAR systems, and

3. map the cross-sections of man-made drainage ditches and perform an accuracy assessment of LiDAR mapping for various surfaces and vegetation coverage.

\section{MOBILE MAPPING SYSTEMS}

Two mobile mapping systems are used in this study: a mediumgrade system, Purdue Wheel-based Mobile Mapping System-High Accuracy (PWMMS-HA), and a high-grade system, Purdue Wheelbased Mobile Mapping System-Ultra High Accuracy (PWMMSUHA). The PWMMS-HA (shown in Figure 1a) is outfitted with four 3D LiDAR units: three Velodyne HDL-32Es and one Velodyne VLP-16 High Resolution. Three FLIR Grasshopper3 9.1MP GigE color cameras are also used for this system. All three cameras utilize an $8 \mathrm{~mm}$ Tamron lens. The remote sensing units of the PWMMS-HA are directly georeferenced by an Applanix POS LV 220 GNSS/INS unit (i.e., the position and orientation information of these units throughout the survey mission are directly derived by the onboard GNSS/INS). The positional accuracy of the POS LV 220 is $\pm 2 \mathrm{~cm}$, and the attitude accuracy is $\pm 0.020 \mathrm{deg}$. The range accuracy of the HDL-32E and VLP-16 are typically $\pm 2 \mathrm{~cm}$ and $\pm 3 \mathrm{~cm}$, respectively. The PWMMS-UHA (shown in Figure $1 b$ ) is equipped with two profiling LiDAR units, a Riegl VUX 1HA and a Z+F Profiler 9012. Two rear-facing FLIR Flea2 FireWire color cameras with $12 \mathrm{~mm}$ lenses are also installed on the PWMMS-UHA. Direct georeferencing of the PWMMS-UHA is provided by a NovAtel ProPak6 GNSS receiver and ISA-100C near navigation grade IMU. Positional accuracy of the NovAtel GNSS/INS system is in the range of \pm 1 to $\pm 4 \mathrm{~cm}$, and the attitude accuracy is $\pm 0.003 \mathrm{deg}$. The range accuracy is $\pm 5 \mathrm{~mm}$ and $\pm 2 \mathrm{~mm}$ for the Riegl and $\mathrm{Z}+\mathrm{F}$ units, respectively.

Camera triggering on the PWMMS-HA is accomplished using the Pulse Per Second (PPS) output of the POS LV as an input to the Grasshopper3's opto-isolated General-Purpose Input/Output (GPIO). In order to achieve a variable frame rate, the PWMMSUHA provides a separate Arduino-based triggering circuit to its two Flea2 cameras. Event feedback for both systems is provided directly from the cameras to the GNSS/INS systems through the strobe feedback GPIO. PointGrey FlyCap is used as the software interface for all cameras on both systems during data collection.

\section{FIELD SURVEY}

The study site is located adjacent to Purdue University's campus in West Lafayette, Indiana. The roadside ditches are present on both sides of the road and covered by a variety of vegetation. The width of the ditches ranges from $2 \mathrm{~m}$ to $10 \mathrm{~m}$, and their depth ranges from $0.2 \mathrm{~m}$ to $1 \mathrm{~m}$. Portions of the surveyed area were recently mowed providing an opportunity to evaluate the impact of vegetation height on mapping accuracy. Figure $2 \mathrm{a}$ is an aerial photo of the study site. Images of location i, ii, iii, and iv, taken by the Grasshopper camera on PWMMS-HA, are shown in Figure $2 \mathrm{~b}$. The MMS datasets were collected on June $13^{\text {th }}, 2019$. The PWMMS-UHA drove first, followed by the PWMMS-HA. The

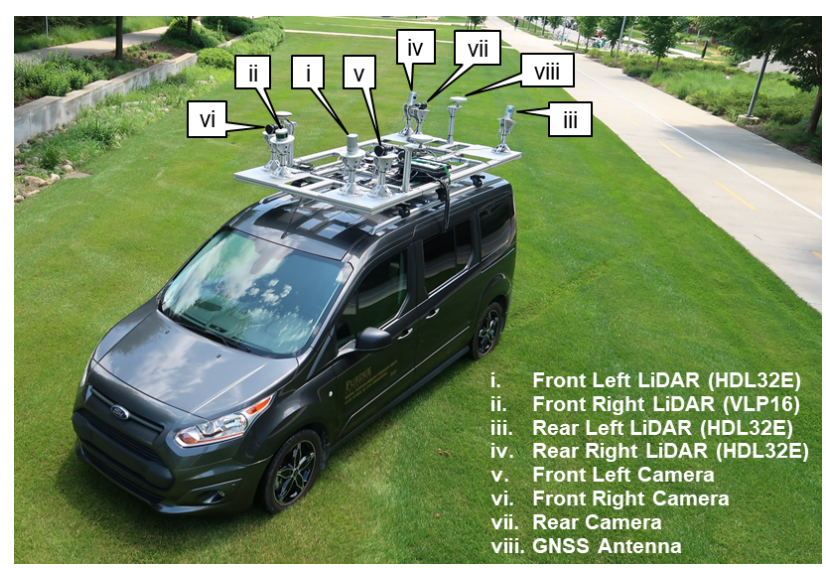

(a) Medium-grade system (PWMMS-HA)

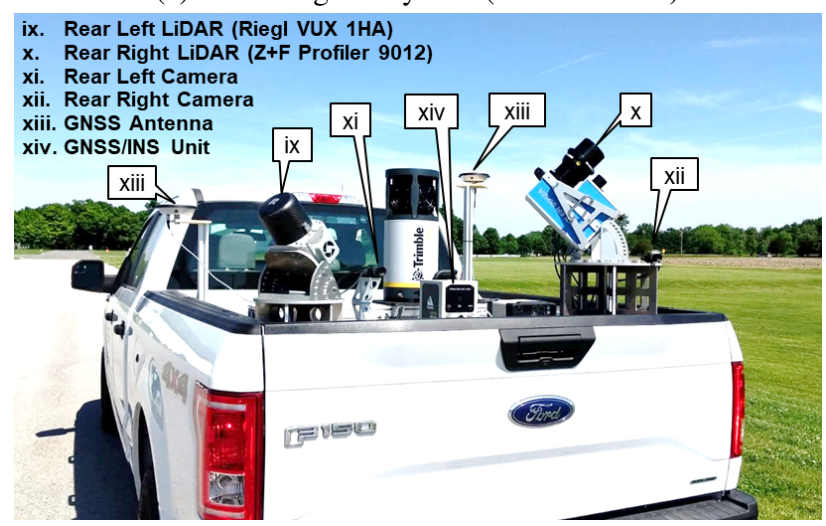

(b) High-grade system (PWMMS-UHA)

Figure 1: Purdue wheel-based mobile mapping systems.

MMSs drove all routes in both directions, so both datasets have two tracks captured at an average driving speed of $30 \mathrm{mph}$ collected over 10 minutes (actual time that the MMSs drove through the study site). An RTK-GNSS survey was carried out on July $15^{\text {th }}, 2019$ to evaluate the geometric quality of the LiDAR-based mapping of the ditches at the four cross-section locations shown in Figure 2a. For each profile, we surveyed a few points on the road, and 20-25 points across the ditch. The team also took few measurements on the sidewalk that was adjacent to the road in profiles i and ii.

\section{METHODOLOGY}

\subsection{Point Cloud Reconstruction}

Raw data collected by the MMS includes LiDAR range and intensity measurements, imagery, and GNSS/INS trajectory as represented by the position and orientation of the vehicle frame. The key to deriving high accuracy point clouds, as well as integrating imagery and LiDAR data, is a system calibration procedure that estimates the relative position and orientation (hereafter, denoted as the mounting parameters) between the GNSS/INS unit and the LiDAR and imaging units. In this study, these mounting parameters are accurately determined through a rigorous calibration procedure (Ravi et al., 2018a; Ravi et al., 2018b). A reconstruction process is then carried out in order to bring all the range measurements from different LiDAR units into one reference coordinate system, which is defined by the reference frame for the GNSS/INS trajectory. By coupling the range and orientation of the laser beams, the position of laser beam footprints relative to 


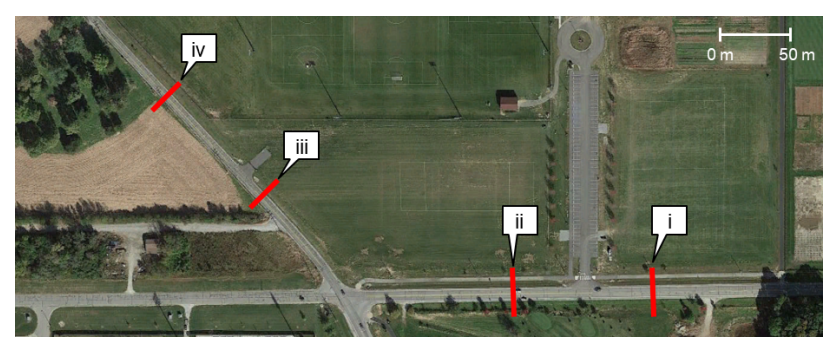

(a) Study site and the surveyed cross-sections (red lines) (aerial photo adapted from a 2018 Google Earth Image)
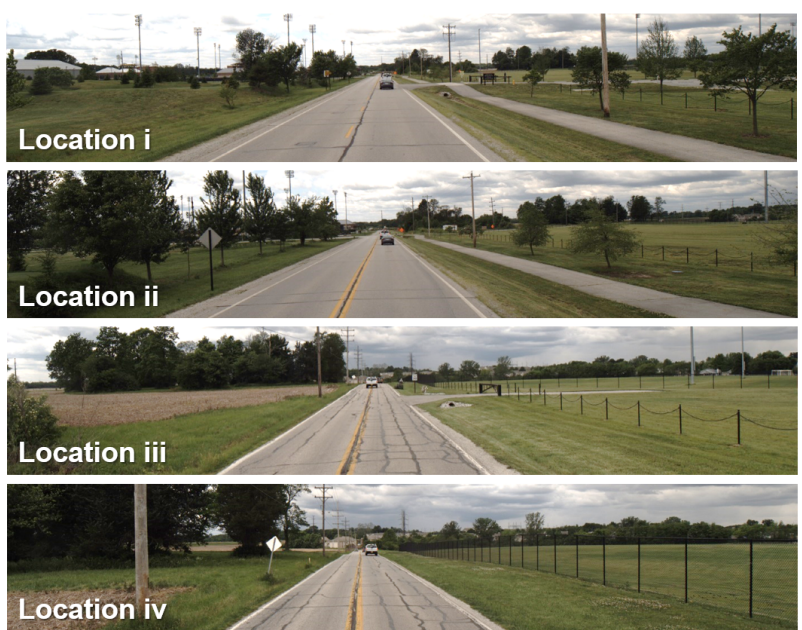

(b) Photo of the surveyed area at location i, ii, iii, and iv

Figure 2: Study site and cross-section locations.

the laser unit frame, $r_{I}^{l u}(t)$, can be obtained. The GNSS/INS integration establishes the position, $r_{v}^{m}(t)$, and orientation, $R_{v}^{m}(t)$ of the vehicle frame relative to the mapping frame. The relationship between the laser unit frame and vehicle frame is expressed by the mounting parameters: lever arm, $r_{l u}^{v}$, and boresight matrix, $R_{l u}^{v}$. The 3D coordinates of a ground point, $I$, in the mapping frame can then be derived through equation (1).

$$
r_{I}^{m}=r_{v}^{m}(t)+R_{v}^{m}(t) r_{l u}^{v}+R_{v}^{m}(t) R_{l u}^{v} r_{I}^{l u}(t)
$$

Apart from point cloud reconstruction, image-LiDAR integration is another equally important advantage of system calibration. Since the mounting parameters between the GNSS/INS unit, LiDAR units, and cameras are accurately determined, all the images are georeferenced, i.e., the camera position and orientation are known in a global reference frame. The images capturing each profile can be identified, and moreover, the LiDAR points can be back-projected to the images. One should note that the image-LiDAR integration benefits the reporting mechanism of ditch mapping (i.e., the ditches can be visualized and reported in $3 \mathrm{D}$ point clouds as well as $2 \mathrm{D}$ images even though they are mainly detected and mapped in 3D space). The image-based visualization is useful for effective mitigation of detected problems during the mapping of ditches (e.g., deviation from the design profile of the ditch, improper grade along the ditch, and debris within the ditch).

\subsection{Profile Extraction and Visualization}

The proposed ditch mapping strategy includes three main aspects: i) geo-referencing, ii) bare earth point cloud extraction, and iii) ditch cross-sectional/longitudinal profile extraction and visualization. All the sensor data are first aligned in a common reference frame so that multi-sensor/multi-date datasets can be effectively fused. The bare earth point cloud representing the terrain is then extracted by a data filtering approach based on cloth simulation, adapted from Zhang et al. (2016). Having the original and the bare earth point clouds, a cross-sectional/longitudinal profile can be extracted at any location. The terrain is represented by line segments connecting the bare earth points. Since all the images are georeferenced, the images capturing each profile can be identified, and the LiDAR points can be back-projected to the images. Therefore, ditches can be visualized and reported in 3D point clouds as well as 2D images even though they are mainly detected and mapped in 3D space.

\section{EXPERIMENTS AND RESULTS}

\subsection{Point Cloud Compatibility Assessment}

In this study, the reconstructed point cloud has an average point density of 6,373 point $/ \mathrm{m}^{2}$ and 4,193 point $/ \mathrm{m}^{2}$, which corresponds to an inter-point spacing of $1.3 \mathrm{~cm}$ and $1.5 \mathrm{~cm}$, for the PWMMSHA and PWMMS-UHA, respectively. The average point density of the PWMMS-HA is higher than the PWMMS-UHA because it has more LiDAR sensors, but the PWMMS-UHA has higher fidelity sensors. One should note that this point density is much higher than that of airborne LiDAR, which is usually less than 10 point $/ \mathrm{m}^{2}$.

The relative vertical accuracy of the data collected by the two MMS data acquisition systems is evaluated by assessing the point cloud compatibility. To assess the compatibility between the derived point clouds from different sensors on a given platform and between different tracks (drive runs) in a given mission, we selected and extracted a segment on the road which is presumed to be planar, and checked the alignment across different sensors/tracks.

- Figure 3a-3g show the PWMMS-HA point cloud alignment along the road of profile i. The red points from westbound and the blue points from eastbound are well-aligned and the noise level (i.e., the spread of the point cloud in the vertical direction) is approximately $0.030 \mathrm{~m}$ and $0.020 \mathrm{~m}$ for HDL 32E and VLP16 LiDAR sensors, respectively (Figure 3b$3 e)$. The point cloud also shows a good alignment across multiple sensors, and the noise level is approximately 0.040 $\mathrm{m}$ for both tracks (Figure $3 \mathrm{f}$ and $3 \mathrm{~g}$ ).

- Figure 3h-3l shows the PWMMS-UHA point cloud alignment within the same area. The result suggests good alignment between two sensors, and the noise level is roughly $0.010 \mathrm{~m}$ and $0.006 \mathrm{~m}$ for Riegl and Z+F, respectively (Figure $3 \mathrm{i}$ and $3 \mathrm{j}$ ). The point clouds across the two tracks are also well-aligned, with a noise level of $0.006 \mathrm{~m}$ (Figure $3 \mathrm{k}$ and 31).

Overall, the point cloud acquired by both systems have valid compatibility in the vertical direction between different tracks for a given sensor, as well as between different sensors for a given track. The PWMMS-UHA has a noise level much lower than the PWMMS-HA.

\subsection{Profile Extraction and Visualization}

The previous section reported the vertical accuracy of the point cloud derived from the PWMMS-HA and the PWMMS-UHA 


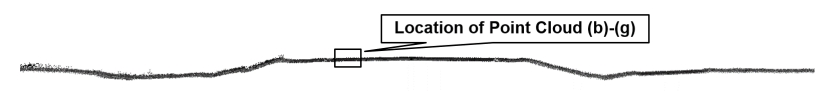

(a) Profile i, PWMMS-HA

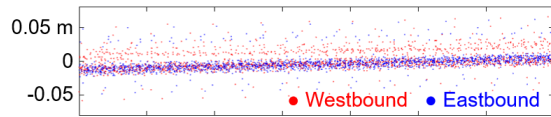

(b) Front HDL32E sensor point cloud

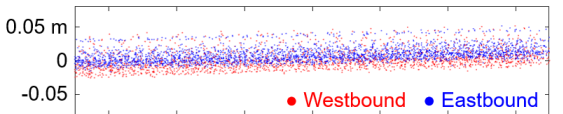

(c) Rear left HDL32E sensor point cloud

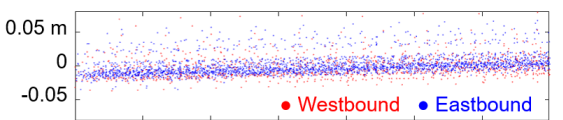

(d) Rear right HDL32E sensor point cloud

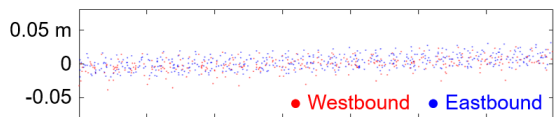

(e) Front VLP16 sensor point cloud

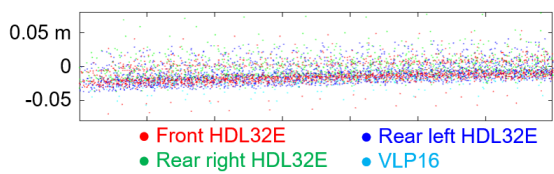

(f) Combined sensor point cloud (Westbound)

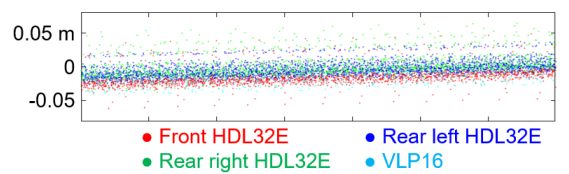

(g) Combined sensor point cloud (Eastbound)

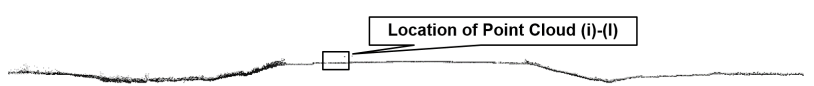

(h) Profile i, PWMMS-UHA

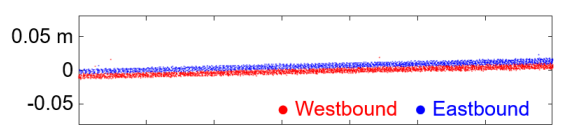

(i) Riegl sensor point cloud

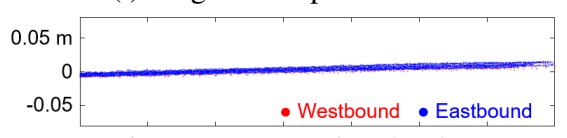

(j) Z+F sensor point cloud

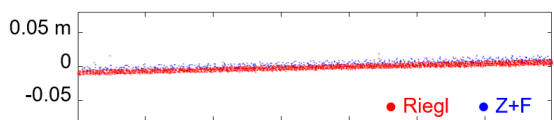

(k) Combined sensor point cloud (Westbound)

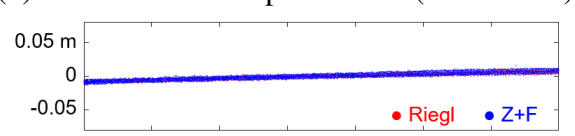

(1) Combined sensor point cloud (Eastbound)

Figure 3: Point cloud compatibility between different sensors and tracks (using profile $\mathrm{i}$ as an example). The units of the horizontal and vertical axes are in meters with one-meter separation between the ticks along the horizontal axis.

was approximately $4 \mathrm{~cm}$ and $1 \mathrm{~cm}$, respectively. With the highaccuracy dense point cloud, mapping a solid surface roadside ditch is not a challenge. However, most of the roadside ditches are covered with vegetation and thus the capability of mapping such ditches as well as identifying the ground surface is critical for evaluation of the system performance. The following analyses evaluate the feasibility of mapping vegetated ditches using MMS-based LiDAR data.

Four ditch cross-sections at location i, ii, iii, and iv in Figure 2 are segmented, reported, and visualized. Figure 4 shows the $3 \mathrm{D}$ and $2 \mathrm{D}$ visualization of the ditch at location iii, where the right side of the road is recently mowed and the left side is unmowed. The cross-sectional profile, bare earth points, terrain, and manually-collected RTK-GNSS survey measurements (hereafter, RTK points) are illustrated in Figure 4(a). The bare earth points are compatible with the RTK points, which indicates that some LiDAR points penetrate to the terrain or close to the terrain while other LiDAR points capture the grass. This evidence shows that LiDAR can penetrate vegetation and report elevations below the visible surface. The degree of penetration depends on the density of the vegetation as well as the size of the laser beam footprint. In addition to $3 \mathrm{D}$ visualization, the cross-sectional profile and the corresponding ground points are projected onto the RGB images. As shown in Figure 4(b), the back-projected points coincide with the corresponding features in the images. The image-based visualization is useful for effective mitigation of detected problems during the mapping of ditches (e.g., deviation from the design profile of the ditch, improper grade along the ditch, and debris within the ditch).

\subsection{Accuracy Assessment}

To quantify the discrepancy between the LiDAR and RTK points, we manually classify the LiDAR/RTK points into four classes: road, sidewalk, mowed grass area, and un-mowed grass area. The elevation difference between each RTK point and the closest LiDAR point is calculated, and the statistics including the mean, standard deviation, median, maximum, minimum, and the rootmean-square error (RMSE) are reported (as shown in Table 1). Figure 5 shows the interquartile range for each class. According to the statistics, the variance of the elevation difference is small for the road and sidewalk, and much larger for mowed and unmowed grass area. The RMSE suggests that the accuracy of ditch mapping is $\pm 2 \mathrm{~cm}$ for PWMMS-HA and $\pm 1 \mathrm{~cm}$ for PWMMSUHA on the road and sidewalk. In the grassy area, the accuracy of ditch mapping is in the range of $\pm 7 \mathrm{~cm}$ and $\pm 11 \mathrm{~cm}$ for mowed and un-mowed areas, respectively. These results show that our MMS units are capable of mapping ditches with a width less than $10 \mathrm{~m}$ and depth less than $1 \mathrm{~m}$. The slightly better accuracy of the PWMMS-HA-based profiles is mainly attributed to better penetration of vegetated surfaces due to its higher point density and larger beam divergence angle of the Velodyne units.

\section{CURRENT AND FUTURE WORK}

The objective of this study is to: 1) visualize the ditch crosssections in $2 \mathrm{D}$ and $3 \mathrm{D}, 2$ ) assess the geometric quality of data collected by two grades of LiDAR-based MMS units, and 3) map the cross-sections of man-made drainage ditches covered with vegetation and evaluate the quality. The compatibility of MMS LiDAR point cloud between different sensors and tracks was validated using a segment of point cloud on the road. The overall point cloud accuracy was $\pm 4 \mathrm{~cm}$ for PWMMS-HA and $\pm 1 \mathrm{~cm}$ for PWMMS-UHA. The ditch mapping result showed that the MMS LiDAR point cloud is capable of mapping a ditch whose width is within $10 \mathrm{~m}$ of the roadway edge and up to $1 \mathrm{~m}$ deep. The mapping accuracy was found to be $\pm 2 \mathrm{~cm}$ (medium-grade system) and $\pm 1 \mathrm{~cm}$ (high-grade system) for solid surface. For rough mowed areas and areas with significant vegetation, the vertical accuracy was found to be $\pm 7 \mathrm{~cm}$ and $\pm 11 \mathrm{~cm}$, respectively, 

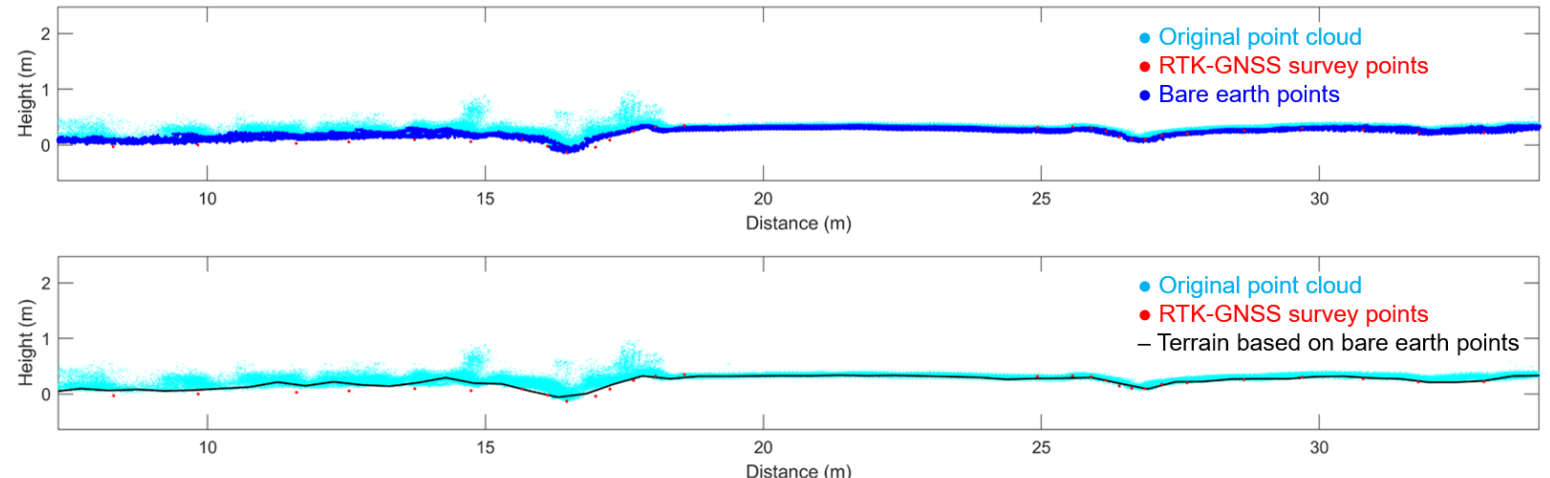

(a) Point cloud, bare earth points, terrain, and RTK-GNSS survey points

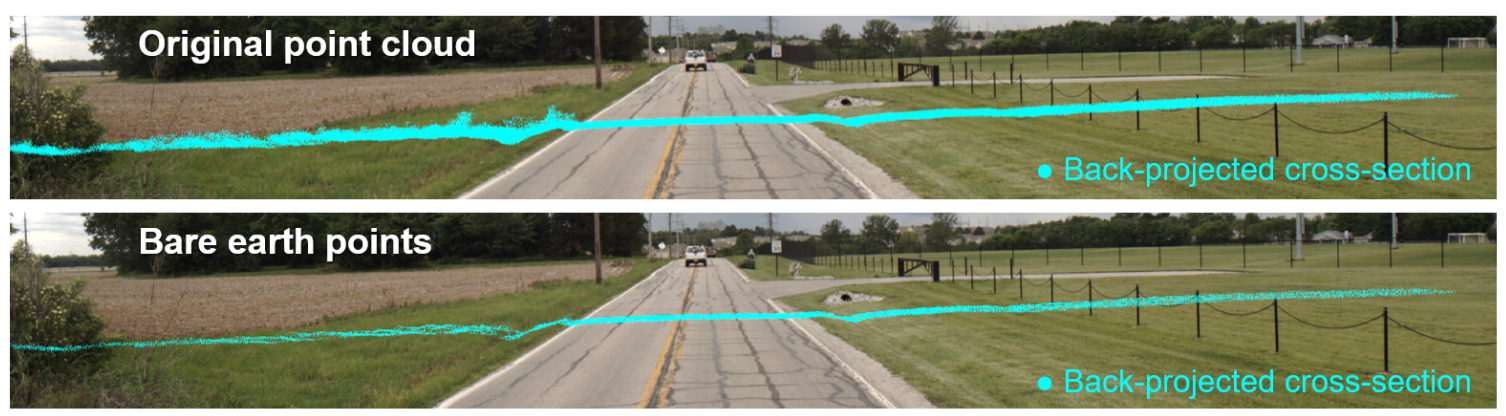

(b) Images with back-projected points

Figure 4: Cross-sectional profile of the ditch at location iii.

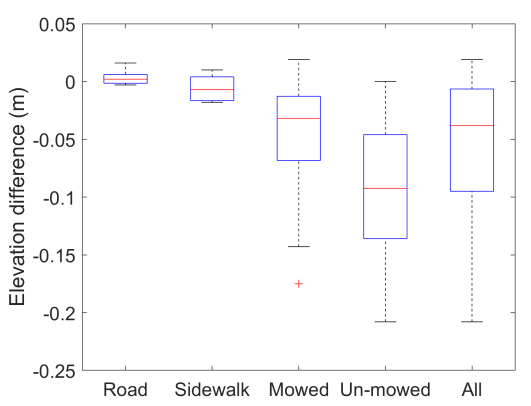

(a) Box and whisker plot for PWMMS-HA

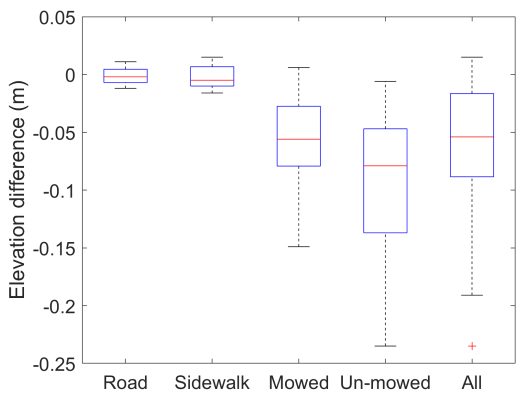

(b) Box and whisker plot for PWMMS-UHA

Figure 5: Characterizing elevation difference between RTK points and LiDAR points for two mobile mapping systems with residual plots of range, $25^{\text {th }}$ percentile, median, and $75^{\text {th }}$ percentile.

for both systems. Current and future work will be focusing on the investigation of the potential of UAV-based LiDAR for map-

\begin{tabular}{|c|c|c|c|c|c|}
\hline & Road & Sidewalk & Mowed & Un-mowed & All \\
\hline \# of points & 12 & 5 & 57 & 34 & 108 \\
\hline \multicolumn{7}{|c|}{ PWMMS-HA } \\
\hline Mean & 0.004 & -0.006 & -0.047 & -0.091 & -0.054 \\
\hline Std. Dev. & 0.007 & 0.012 & 0.045 & 0.057 & 0.055 \\
\hline RMSE & 0.007 & 0.012 & 0.065 & 0.107 & 0.077 \\
\hline Median & 0.002 & -0.007 & -0.032 & -0.092 & -0.038 \\
\hline Max. & 0.016 & 0.010 & 0.019 & 0.000 & 0.019 \\
\hline Min. & -0.003 & -0.018 & -0.175 & -0.208 & -0.208 \\
\hline \multicolumn{7}{|c|}{ PWMMS-UHA } \\
\hline Mean & -0.001 & -0.002 & -0.059 & -0.094 & -0.061 \\
\hline Std. Dev. & 0.007 & 0.012 & 0.039 & 0.059 & 0.053 \\
\hline RMSE & 0.007 & 0.011 & 0.071 & 0.110 & 0.080 \\
\hline Median & -0.002 & -0.005 & -0.056 & -0.079 & -0.054 \\
\hline Max. & 0.011 & 0.015 & 0.006 & -0.006 & 0.015 \\
\hline Min. & -0.012 & -0.016 & -0.149 & -0.235 & -0.235 \\
\hline
\end{tabular}

Table 1: Statistics of the elevation difference between RTK points and LiDAR points (unit: $\mathrm{m}$ ) for the four cross-sections shown in Figure 2a.

ping roadside ditches, and automated extraction and reporting of information pertaining to the mapped ditches.

\section{ACKNOWLEDGEMENTS}

This work was supported in part by the Joint Transportation Research Program administered by the Indiana Department of Transportation and Purdue University.

The authors would like to thank the Digital Photogrammetry Research Group (DPRG) members, Yi-Ting Cheng, Seyyed Meghdad Hasheminasab, Tian Zhou, Radhika Ravi, and Evan Flatt, for their help throughout the data collections.

The contents of this paper reflect the views of the authors, who 
are responsible for the facts and the accuracy of the data presented herein, and do not necessarily reflect the official views or policies of the sponsoring organizations. These contents do not constitute a standard, specification, or regulation.

\section{REFERENCES}

Buchanan, B., Easton, Z. M., Schneider, R. L., Walter, M. T. (2013). Modeling the hydrologic effects of roadside ditch networks on receiving waters. Journal of hydrology, 486, 293-305.

Schneider, R., Orr, D., Johnson, A. (2019). Understanding Ditch Maintenance Decisions of Local Highway Agencies for Improved Water Resources across New York State. Transportation Research Record. https://doi.org/10.1177/0361198119854092

Matos, J. A. (2016). Improving Roadside Ditch Maintenance Practices in Ohio (Doctoral dissertation, University of Cincinnati).

Levavasseur, F., Lagacherie, P., Bailly, J. S., Biarnès, A., Colin, F. (2015). Spatial modeling of man-made drainage density of agricultural landscapes. Journal of Land Use Science, 10(3), 256276.

Rapinel, S., Hubert-Moy, L., Clément, B., Nabucet, J., Cudennec, C. (2015). Ditch network extraction and hydrogeomorphological characterization using LiDAR-derived DTM in wetlands. Hydrology research, 46(2), 276-290.

Ibeh, C., Pallai, C., Saavedra, D. (2017). Lidar-based roadside ditch mapping in York and Lancaster Counties, Pennsylvania.

Roelens, J., Höfle, B., Dondeyne, S., Van Orshoven, J., Diels, J. (2018a). Drainage ditch extraction from airborne LiDAR point clouds. ISPRS journal of photogrammetry and remote sensing, 146, 409-420.

Roelens, J., Rosier, I., Dondeyne, S., Van Orshoven, J., Diels, J. (2018b). Extracting drainage networks and their connectivity using LiDAR data. Hydrological processes, 32(8), 1026-1037.

Ravi, R., Lin, Y. J., Elbahnasawy, M., Shamseldin, T., Habib, A. (2018a). Simultaneous System Calibration of a Multi-LiDAR Multicamera Mobile Mapping Platform. IEEE Journal of Selected Topics in Applied Earth Observations and Remote Sensing, 11(5), 1694-1714. doi: 10.1109/JSTARS.2018.2812796

Ravi, R., Lin, Y. J., Elbahnasawy, M., Shamseldin, T., Habib, A. (2018b). Bias Impact Analysis and Calibration of Terrestrial Mobile LiDAR System With Several Spinning Multibeam Laser Scanners. IEEE Transactions on Geoscience and Remote Sensing. doi: 10.1109/TGRS.2018.2812782

Zhang, W., Qi, J., Wan, P., Wang, H., Xie, D., Wang, X., Yan, G. (2016). An easy-to-use airborne LiDAR data filtering method based on cloth simulation. Remote Sensing, 8(6), 501. 